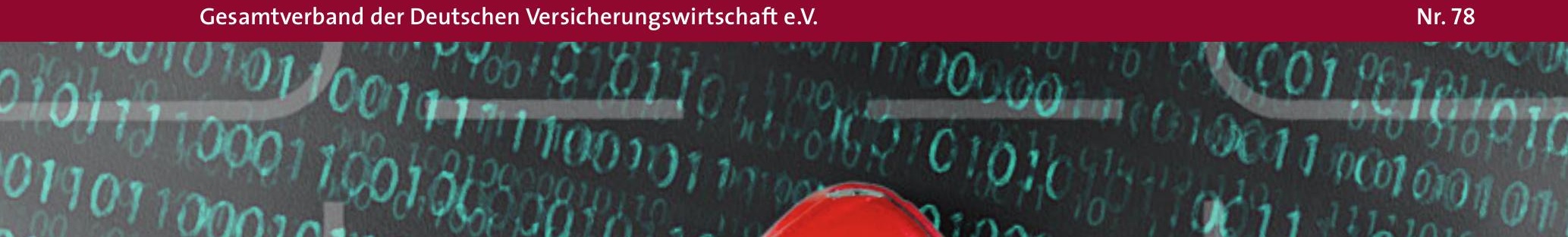

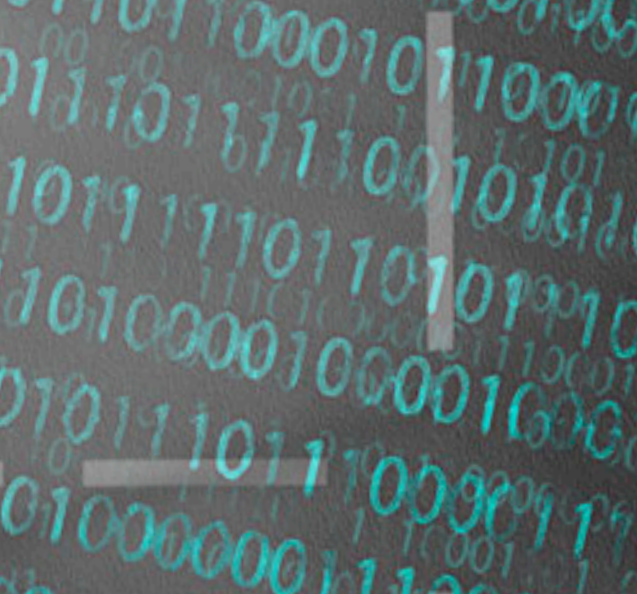

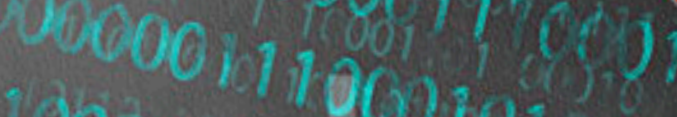

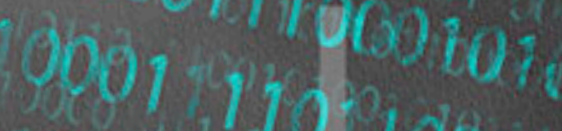
$10.7,000,1,9000$ $1: 1,000912$

\title{
Erhöht automatisiertes Fahren die Sicherheit? \\ Unfallforschung kompakt
}



Inhalt

Einleitung

Einteilung und Abgrenzung

moderner Assistenz- und

Automatisierungsfunktionen

Einfluß auf die Verkehrssicherheit

Grenzen: Der Mensch im Mittelpunkt

Technische Anforderungen an

Automatisierungsfunktionen

Fazit 


\section{Einleitung}

Automatisiertes Fahren wird als Zukunft der Mobilität gesehen. Es wird erwartet, dass dadurch die Leistungsfähigkeit des Verkehrs gesteigert und die Zahl der Straßenverkehrsopfer reduziert wird sowie Emissionen und Staus zurückgehen werden. Dies wird mehr ein mehrdimensionaler, schrittweiser Übergangsprozess als ein schneller Wandel sein.

Die neue Technologie wird dabei sowohl im Pkw als auch im Nutzfahrzeug Einzug halten. Nach jetzigem Stand der Technik würde es sich bei den Fahrzeugen um Level 2oder Level 3-Fahrzeuge handeln, deren automatisierte Fahrfunktion typischerweise nur auf Autobahnen aktiv ist [1]. Mit der Weiterentwicklung der Technologie werden Fahrzeuge mit Fahrfunktionen höherer Automatisierungsstufen und in weiteren Anwendungsbereichen neben der Autobahn schrittweise marktreif. Etwas anders sieht es bei Parkfunktionen aus. Hier könnte die Entwicklung schneller hin zu vollautomatisierten Funktionen verlaufen.

Mit Stand heute wird der Verkehr in absehbarer Zukunft durch das Nebeneinander von Fahrzeugen unterschiedlicher Automatisierungsgrade und manuell gesteuerter Fahrzeuge geprägt sein. Diese Entwicklung wird sowohl Pkw als auch Nutzfahrzeuge betreffen. 
Einteilung und Abgrenzung moderner Assistenz- und Automatisierungsfunktionen

\section{Einteilung und Abgrenzung moderner Assistenz- und Automatisierungsfunktionen}

Die Diskussion um automatisiertes Fahren setzt ein klares Verständnis über die Eigenschaften und Fähigkeiten der Funktionen voraus.

Die Fahraufgabe lässt sich in die Navigations-, Bahnführungs- und Stabilisierungsebene einteilen [2]. Entsprechend dieses Modells beschreibt die Navigationsebene die Routenplanung, die Bahnführungsebene den Soll/ Ist-Abgleich durch den Fahrer - mithin das dynamische Fahren - und die Stabilisierungsebene die Kontrolle der Regelabweichungen im geschlossenen Regelkreis.
Assistenz- und Automatisierungsfunktionen wirken auf der Bahnführungsebene. Hier kann man in drei unterschiedliche Wirkweisen unterscheiden [3]. Dabei handelt es sich um die informierenden und warnenden Funktionen, die kontinuierlich automatisierenden Funktionen und die temporär intervenierenden Systeme (siehe Tab.1).

Dieser Ansatz hat den Vorteil, dass Fahrerassistenzsysteme und automatisierte Fahrfunktionen unterscheidbar werden. Selbst innerhalb der Fahrerassistenzsysteme werden Unterschiede sichtbar, die sich klar aus ihrer Wirkweise ableiten lassen. Die Wirkweise B beschreibt die in der Diskussion befindlichen Automatisierungs-stufen (siehe Tabelle 2). Level 1 beschreibt dabei nur bestimmte Fahrerassistenzsysteme, die die Längs- und Querführung regeln. Das sind der Abstandregeltempomat und der Spurhalteassistent. Der Spurverlassenswarner ist dagegen konsequenterweise der Wirkweise A/2 zuzuordnen.

Tab. 1: Assistenz- und Automatisierungsfunktionen auf der Bahnführungsebene [3]

\begin{tabular}{|c|c|c|}
\hline $\begin{array}{l}\text { Wirkweise } A \\
\text { Informierende und warnende } \\
\text { Funktionen }\end{array}$ & $\begin{array}{c}\text { Wirkweise B } \\
\text { Kontinuierlich automatisierende } \\
\text { Funktionen }\end{array}$ & $\begin{array}{c}\text { Wirkweise C } \\
\text { In unfallgeneigten Situationen } \\
\text { temporär intervenierende Systeme }\end{array}$ \\
\hline $\begin{array}{l}\text { Wirken ausschließlich und } \\
\text { „mittelbar“ über den Fahrer: } \\
\text { 1. Statusinformationen, } \\
\text { z. B. Verkehrszeichenerkennung } \\
\text { 2. Abstrakte Warnung, } \\
\text { z. B. Spurverlassenswarner } \\
\text { 3. Konkrete Warnung, z. B. } \\
\text { - Totwinkelwarner oder } \\
\text { - Auffahrwarner }\end{array}$ & $\begin{array}{l}\text { Haben unmittelbaren Einfluss } \\
\text { auf die Fahrzeugsteuerung, } \\
\text { sind immer übersteuerbar. } \\
\text { Definition nach SAE J3016 [1] bzw. } \\
\text { VDA/BASt [4] }\end{array}$ & $\begin{array}{l}\text { Präventive maschinelle } \\
\text { Intervention bei negativer } \\
\text { Situationsprognose, z. B. } \\
\text { - Notbremsassistent } \\
\text { - Ausweichassistent }\end{array}$ \\
\hline
\end{tabular}


Einteilung und Abgrenzung moderner Assistenz- und Automatisierungsfunktionen

Tab. 2: Stufen der Automatisierung nach SAE J3016 und VDA/BASt [1] [4]

\begin{tabular}{|c|c|}
\hline Nomenklatur & $\begin{array}{l}\text { Fahraufgaben des Fahrers } \\
\text { nach Automatisierungsgrad }\end{array}$ \\
\hline $\begin{array}{l}\text { Fahrerlos } \\
\text { Full Automation } \\
\text { Level } 5\end{array}$ & $\begin{array}{l}\text { Das System übernimmt die Fahraufgabe vollumfänglich bei allen Straßentypen, } \\
\text { Geschwindigkeitsbereichen und Umgebungsbedingungen }\end{array}$ \\
\hline $\begin{array}{l}\text { Vollautomatisiert } \\
\text { High Automation } \\
\text { Level } 4\end{array}$ & $\begin{array}{l}\text { Das System übernimmt Quer- und Längsführung vollständig in einem } \\
\text { definierten Anwendungsfall }\end{array}$ \\
\hline $\begin{array}{l}\text { Hochautomatisiert } \\
\text { Conditional Automation } \\
\text { Level } 3\end{array}$ & $\begin{array}{l}\text { Das System übernimmt Quer- und Längsführung für einen gewissen Zeitraum } \\
\text { in spezifischen Situationen }\end{array}$ \\
\hline $\begin{array}{l}\text { Teilautomatisiert } \\
\text { Partial Automation } \\
\text { Level } 2\end{array}$ & $\begin{array}{l}\text { Das System übernimmt Quer- und Längsführung (für einen gewissen Zeitraum } \\
\text { oder/und in spezifischen Situationen) }\end{array}$ \\
\hline $\begin{array}{l}\text { Assistiert } \\
\text { Driver Assistance } \\
\text { Level } 1\end{array}$ & $\begin{array}{l}\text { Fahrer führt dauerhaft entweder die Quer- oder die Längsführung aus. } \\
\text { Die jeweils andere Fahraufgabe wird in gewissen Grenzen vom System ausgeführt. } \\
\text { z. B. ACC, Spurhalteassistent }\end{array}$ \\
\hline $\begin{array}{l}\text { Fahrer } \\
\text { No Automation } \\
\text { Level o }\end{array}$ & $\begin{array}{l}\text { Fahrer führt dauerhaft (während der gesamten Fahrt) die Längsführung } \\
\text { (Beschleunigen/Verzögern) und die Querführung (Lenken) aus. }\end{array}$ \\
\hline
\end{tabular}




\section{Einfluß auf die Verkehrssicherheit}

Im Jahr 2017 starben auf deutschen Straßen 3.177 Menschen und 388.200 Personen wurden verletzt [5]. Für das Jahr 2016 sind die Getöteten nach der Art der Verkehrsteilnahme in der folgenden Abbildung 1 dargestellt.

Getötete nach Art der Verkehrsteilnahme im Jahr 2016 $n=3.206$

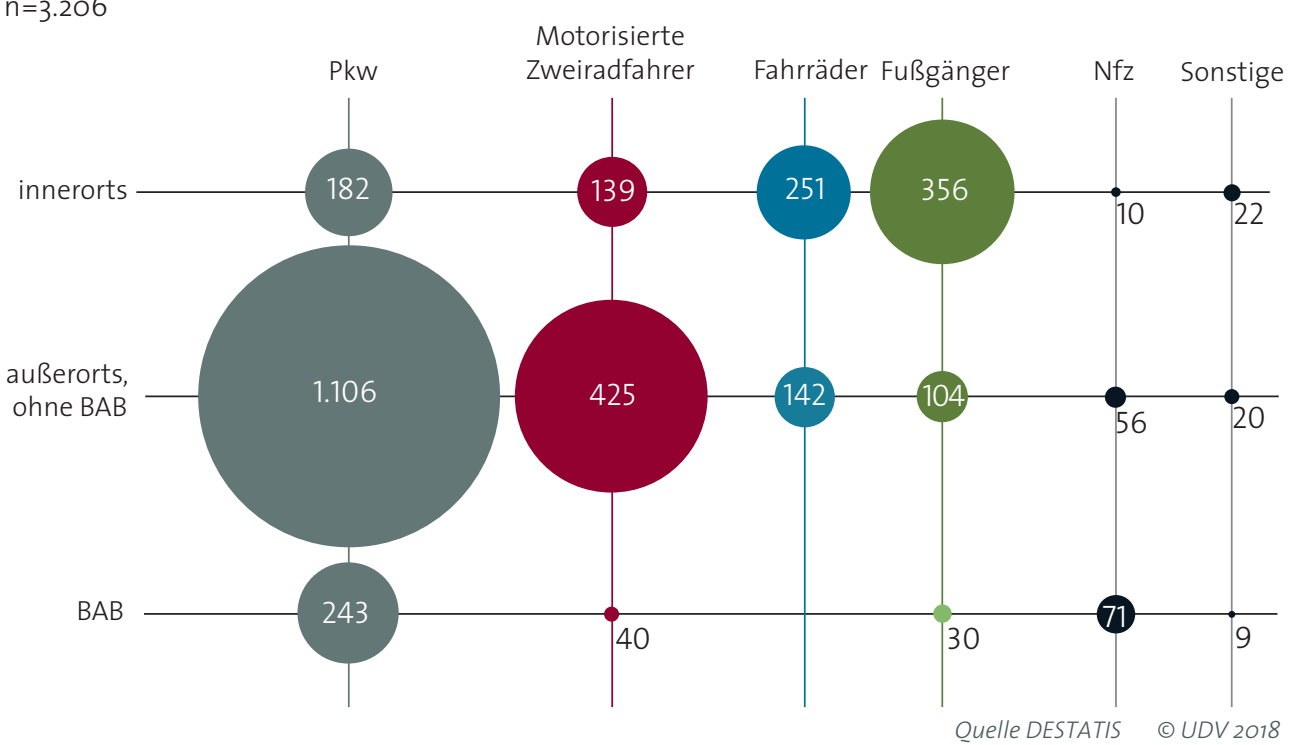

Abb. 1: Getötete nach Art der Verkehrsteilnahme in Deutschland im Jahr 2016 [6]

Es wird deutlich, dass die Schwerpunkte im Unfallgeschehen mit Getöteten für Pkw im Außerortsbereich liegen. Vor allem Unfälle durch das Abkommen von der Fahrbahn und der Kollision mit Bäumen bilden hier einen Schwerpunkt. Unfälle mit motorisierten Zweirädern außerorts, und hier vor allem mit Motorrädern, stehen an zweiter Stelle des Unfallgeschehens. Die dritte Problemgruppe bilden die ungeschützten Verkehrsteilnehmer bei Unfällen innerorts. Hier werden vor allem Fußgänger und Radfahrer bei Kollisionen mit Pkw getötet. 
Einfluß auf die Verkehrssicherheit

\section{Fahrerassistenzsysteme}

Neue Technologien sollen zum Ziel haben, gerade an den Schwerpunkten des Unfallgeschehens anzusetzen und ihre positive Wirkung zu entfalten. Dem entsprechen die folgenden Berechnungen in Tabelle 3. Der Notbremsassistent ist von sehr großer Bedeutung für den Pkw-Unfall. Er adressiert häufige Unfallszenarien wie den Längsverkehrsunfall mit 22 Prozent und auch Teile des Kreuzungsunfalls. Dabei wären fast 20 Prozent aller Pkw-Unfälle durch einen Notbremsassistenten vermeidbar, der vorausfahrende und stehende zweispurige Fahrzeuge erkennen kann.
Erkennt der Notbremsassistent noch zusätzlich Fußgänger, wäre er in der Lage, 24,5 Prozent aller Pkw-Unfälle zu verhindern. Die Verbraucherschutzorganisation Euro NCAP honoriert diese Systeme seit 2016 in ihrer Fahrzeugsicherheitsbewertung [8]. Eine gesetzliche Vorschrift zur standardmäßigen Ausrüstung aller Pkw mit diesen wertvollen Sicherheitssystemen ist angedacht und soll bis etwa zur Mitte des nächsten Jahrzehnts umgesetzt werden [9].

Handlungsbedarf besteht vor allem noch bei Kreuzungssituationen, die mit etwa einem Drittel den Hauptteil des Pkw-Unfallgeschehens ausmachen. Ab 2020 soll ein

Tab. 3: Unfallszenarien und Sicherheitspotenzial von Fahrerassistenzsystemen für Pkw [7]

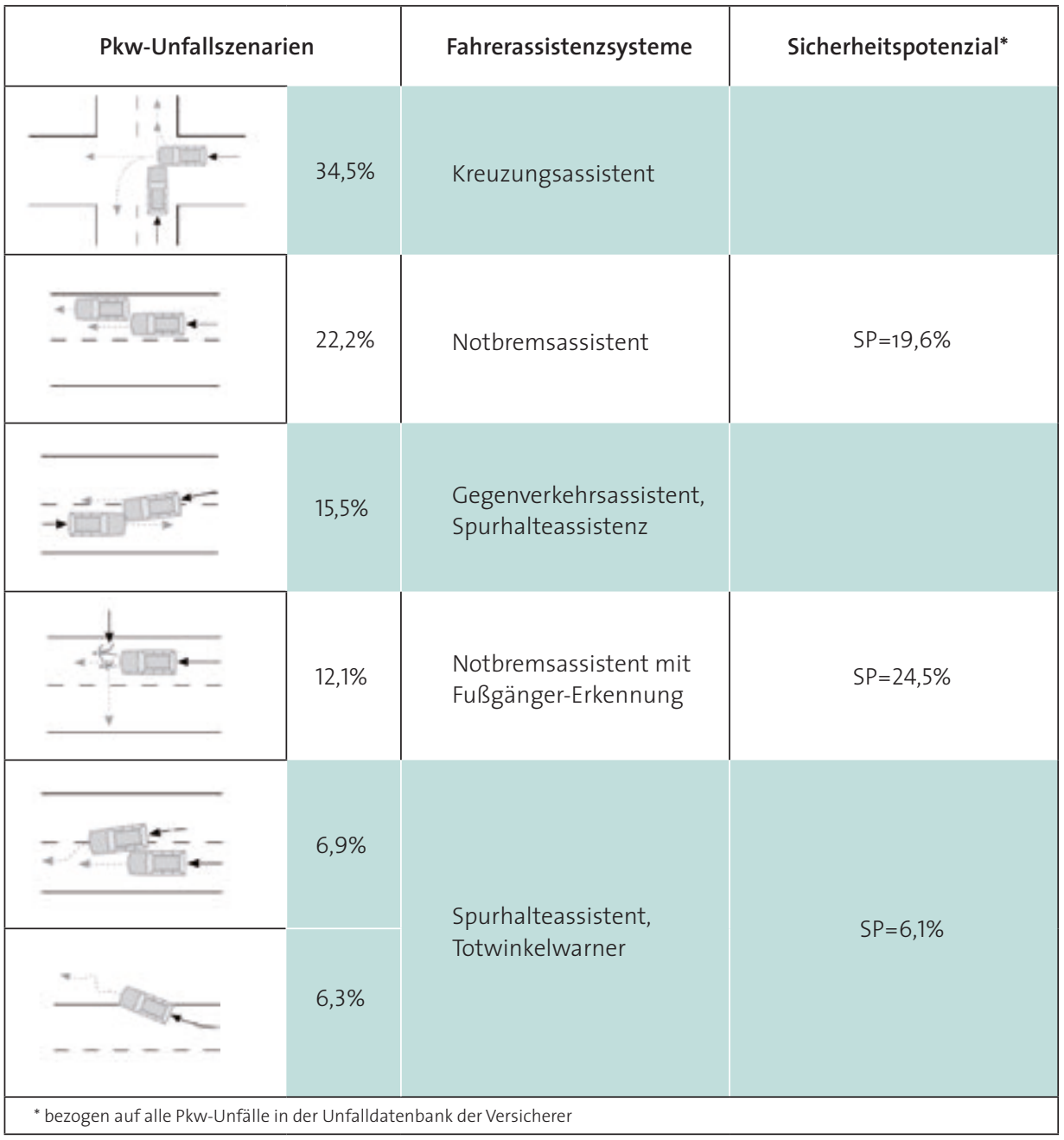


Einfluß auf die Verkehrssicherheit

System, das Unfälle mit kreuzenden Fahrzeugen adressiert, bei Euro NCAP honoriert werden [8]. Spätestens dann werden diese Systeme vermehrt auf den europäischen Markt kommen und ihre positive Wirkung im Unfallgeschehen entfalten können.

\section{Automatisierungsfunktionen}

Auf Basis der Unfalldatenbank der Versicherer (UDB) führen erste Ableitungen mit entsprechenden Annahmen zu dem in der Abbildung 2 dargestellten Verkehrssicherheitspotential einer generischen hochautomatisierten Fahrfunktion (HAF) für Pkw und Lkw [8]. Die vereinfachte Annahme dieser Betrachtung ist, dass sich eine automatisierte Fahrfunktion in erster Linie durch die Addition des Sicherheitspotentials der Notbremse (AEB), des Spurhalteassistenten (LKA) und des Totwinkelwarners (LCA) auszeichnet. Hierbei noch nicht berücksichtigt sind denkbare und in einer Übergangsphase des Mischverkehrs, bestehend aus automatisierten und herkömmlichen Fahrzeugen, auch wahrscheinlich eintretende negative Effekte der automatisierten Fahrfunktion im Unfallgeschehen, da sie noch nicht quantifizierbar sind.

Deutlich wird, dass die Verkehrssicherheit von einer automatisierten Fahrfunktion für Pkw auf Autobahnen nur gering profitieren würde (4,5\% aller Pkw-Unfälle). Dagegen würde diese Funktion für Lkw auf Autobahnen einen deutlichen Mehrwert liefern. Hier könnten 14,5 Prozent aller Unfälle mit Lkw-Beteiligung positiv beeinflusst werden. Auf der anderen Seite zeigt sich, dass eine automatisierte Fahrfunktion für Pkw mit etwa 33 Prozent positiv beeinflussbarer Unfälle vor allem innerorts die Verkehrssicherheit verbessern könnte. Allerdings sind die Anforderungen an die Technologie aufgrund der Komplexität des innerörtlichen Verkehrsgeschehens deutlich höher als auf Autobahnen, so dass die ersten Anwendungen in naher Zukunft im Pkw die Autobahnfahrt adressieren werden.
Abb. 2: Einfluss der Hochautomatisierten Fahrfunktion (HAF) auf das Unfallgeschehen in Deutschland [10] 
Einfluß auf die Verkehrssicherheit

Den Schadenaufwand der Versicherer bis 2035 analysiert eine Studie des GDV [11]. Es wird u.a. deutlich, dass viele, durch die Versicherer regulierte, Schäden durch Fahrerassistenzsysteme und automatisierte Fahrfunktionen auch zukünftig nicht vermieden werden können (Abb. 3). dem Ergebnis, dass selbst bei schneller Verbreitung von Fahrerassistenzsystemen und automatisierten Fahrfunktionen die Entschädigungsleistungen der Kfz-Versicherer bis 2035 nur um etwa $15 \%$ reduziert sein werden. Der Mensch verursacht im Mittel nur alle drei Millionen

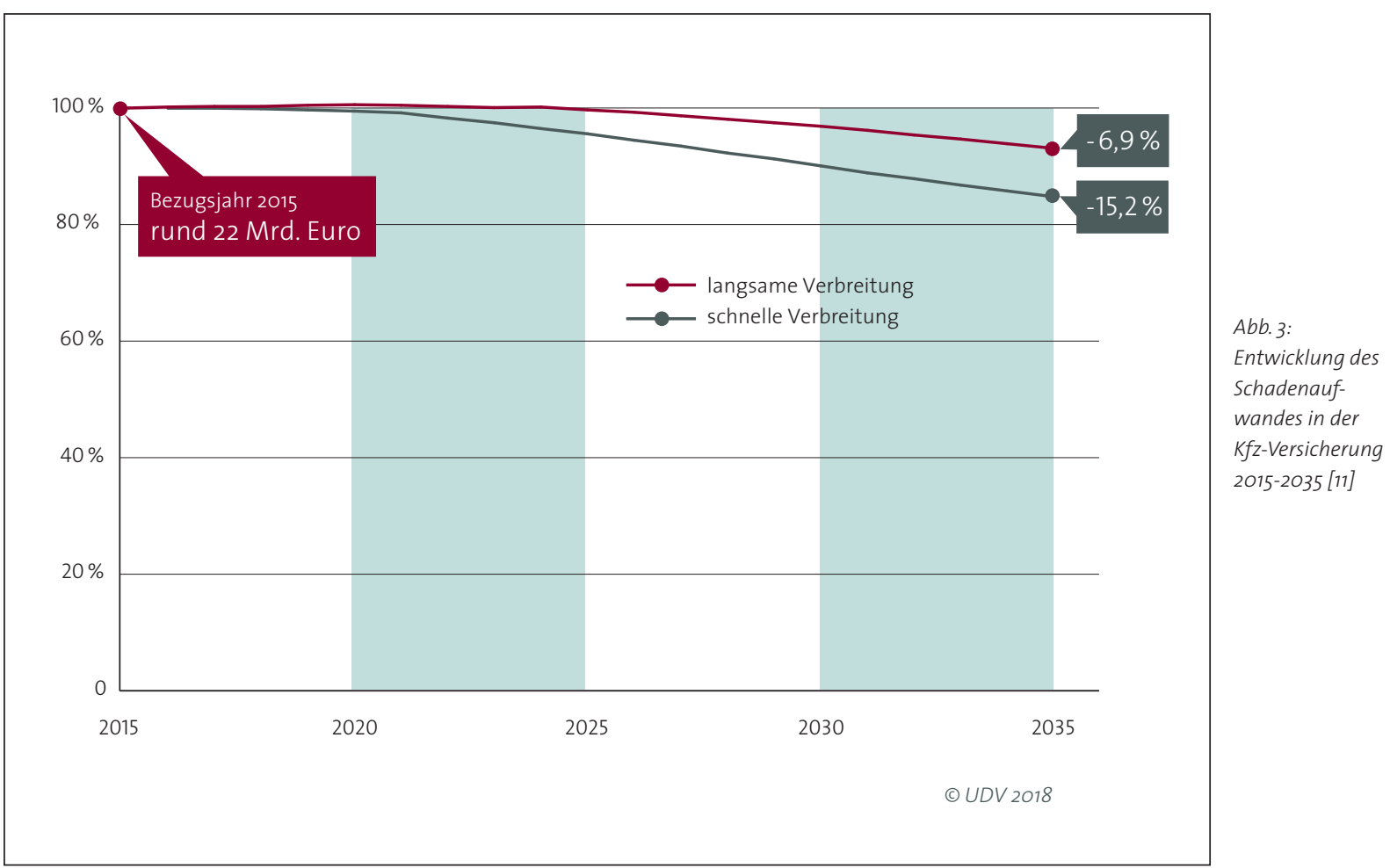

Dazu zählen z. B. Diebstahl, Steinschlag, Hagel oder Marderbiss. Die neue Technologie führt auch zu erhöhten Reparaturkosten, da zusätzliche Sensorik am Fahrzeug verbaut ist. Ein grundsätzlicher Punkt betrifft sowohl Fahrerassistenzsysteme als auch automatisierte Fahrfunktionen bei der Verbreitung der Technik in der Fahrzeugflotte. Diese neuen Systeme werden zu Beginn nur in ausgewählten Neuwagen der gehobenen Fahrzeugklassen angeboten werden. Dazu kommt, dass das durchschnittliche Fahrzeugalter in Deutschland immer weiter steigt. All dies wird dazu führen, dass die Systeme erst lange nach ihrer Markteinführung in nahezu allen Fahrzeugen vorhanden sein werden und somit der positive Effekt auf die Verkehrssicherheit erst deutlich verzögert wirken kann. Auch deshalb kommt die Studie zu
Kilometer einen Unfall mit Personenschaden [12]. Das entscheidende Kriterium ist, dass die diskutierten automatisierten Systeme mindestens den Grad an Sicherheit erreichen, wie die heutigen, durch den Fahrer gesteuerten, Systeme. Der Sicherheitsnachweis sollte mit Hilfe eines transparenten Verfahrens, das auch numerische Methoden beinhalten kann, nachvollziehbar dargelegt werden.

Die Verkehrssicherheit muss von dieser technologischen Entwicklung profitieren. Allerdings bedarf dies einer langfristigen Strategie. Denn zum einen werden erste Anwendungen wie der Stau-Pilot oder der Autobahn-Pilot nur auf der Autobahn verfügbar sein. Zum anderen wird die Durchdringung dieser Technologien in der Fahrzeugflotte einen langen Zeitraum in Anspruch nehmen. 


\section{Grenzen:}

\section{Der Mensch im Mittelpunkt}

In naher Zukunft ist im Rahmen der Entwicklungsstufen des teil- und hochautomatisierten Fahrens noch eine Kontrolle durch den Fahrer mit der Möglichkeit zum jederzeitigen Eingreifen in Problemsituationen vorgesehen. Die Ausübung dieser Kontrolle soll dem Fahrer möglich sein, obwohl zumindest die Gefahr besteht, dass ihm die Grenzen der Funktionalität nicht bekannt sind und diese sich auch von Fahrzeug zu Fahrzeug unterscheiden. Die Fahrer werden klar verstehen müssen, was ihre Fahrzeuge leisten können und wo ihre Verantwortung liegt.

Es ist unwahrscheinlich, dass sich die Verkehrsinfrastruktur kurzfristig anpassen wird, um die Umwelt für selbstfahrende Fahrzeuge einfacher zu gestalten. Vielmehr müssen die Fahrzeuge sehr viel leistungsfähiger werden, um auf allen Straßen selbstständig fahren zu können. Entwickler und Forscher gehen allerdings auch davon aus, dass es zukünftig in Städten sogenannte Automatisierungszonen geben wird, in denen Fahrzeuge überwacht und kooperativ Waren oder Personen autonom transportiert werden [13].

Inzwischen sind erste Fahrzeuge erhältlich, mit denen teilautomatisiert gefahren werden kann. Der Fahrer muss hier während der Aktivierung der Funktion aufmerksam sein und das System ständig überwachen. Langjährige Forschung in verschiedenen Disziplinen zeigt, dass Menschen derartige Überwachungsaufgaben nicht fehlerfrei ausführen können. Daraus könnten neue Unfallmuster mit Beteiligung dieser Fahrzeuge resultieren. Erschwerend kommt der Umstand hinzu, dass moderne Fahrzeuge unterschiedliche Fahrfunktionen verschiedener Automatisierungsstufen anbieten werden. Hier drängen sich Funktionen auf, die sich auch in ihren Anwendungsbereichen deutlich unterscheiden: So werden Fahrzeuge hoch- oder vollautomatisierte Parkfunktionen anbieten und gleichzeitig nur teilauto- matisierte Fahrfunktionen auf Autobahnen bereitstellen. Weniger offensichtlich und damit potentiell problematischer für den Fahrer sind Funktionen, die im selben Anwendungsbereich liegen. Ein Fahrzeug, das bei idealen Bedingungen in der Lage ist, eine automatisierte Autobahnfahrt im Level 4 anzubieten, kann bei schlechter werdenden Umgebungsbedingungen nur noch eine teilautomatisierte Längs- und Querführung offerieren. Entscheidend wird in diesem Fall sein, dass der Fahrer die unterschiedlichen Systemgrenzen und seinen Verantwortungsbereich verstanden hat. Idealerweise sollten Übergänge zwischen assistierten und automatisierten Fahrfunktionen durch eine eindeutige MenschMaschine-Schnittstelle im Fahrzeug umgesetzt sein. So bietet es sich an, das Lenkrad für automatisierte Fahrfunktionen als neue optische Schnittstelle zu nutzen. Nur wenn das Fahrzeug die Verantwortung übernimmt, wird dies das Lenkrad durch einen eindeutigen Farbwechsel mitteilen. Bei assistierten Fahrfunktionen in denen der Fahrer die Verantwortung hat, soll das Lenkrad - wie in heutigen Fahrzeugen auch - keine Informationen tragen.

In den nächsten Jahren werden dann Systeme verfügbar sein, die für bestimmte Zeiträume selbständig fahren, aber wiederum nur ausgewählte Situationen bewältigen können. Man bezeichnet dies als hochautomatisiertes Fahren. Hier muss der Fahrer das Fahrzeug zwar nicht mehr die ganze Zeit überwachen, aber bei Erreichen der Systemgrenzen nach Aufforderung eingreifen.

Das hier beschriebene grundsätzliche Problem des dauerhaften Überwachens und des Eingreifens in kritischen Situationen gründet in einer menschlichen Eigenschaft, die Psychologen bereits vor mehr als 100 Jahren untersucht haben [14]. Das daraus entstandene Yerkes-Dodson-Gesetz beschreibt den allgemeingültigen Zusammenhang zwischen der Leistungsfähigkeit und dem Erregungszustand eines Menschen. Dabei befindet sich die Leistungsfähigkeit bei geringer Erregung auf einem Minimum, um bei steigender Erregung ein Maximum zu durchlaufen. Bei nun steigender Erregung fällt die Leistungsfähigkeit jedoch wieder ab, um dann 


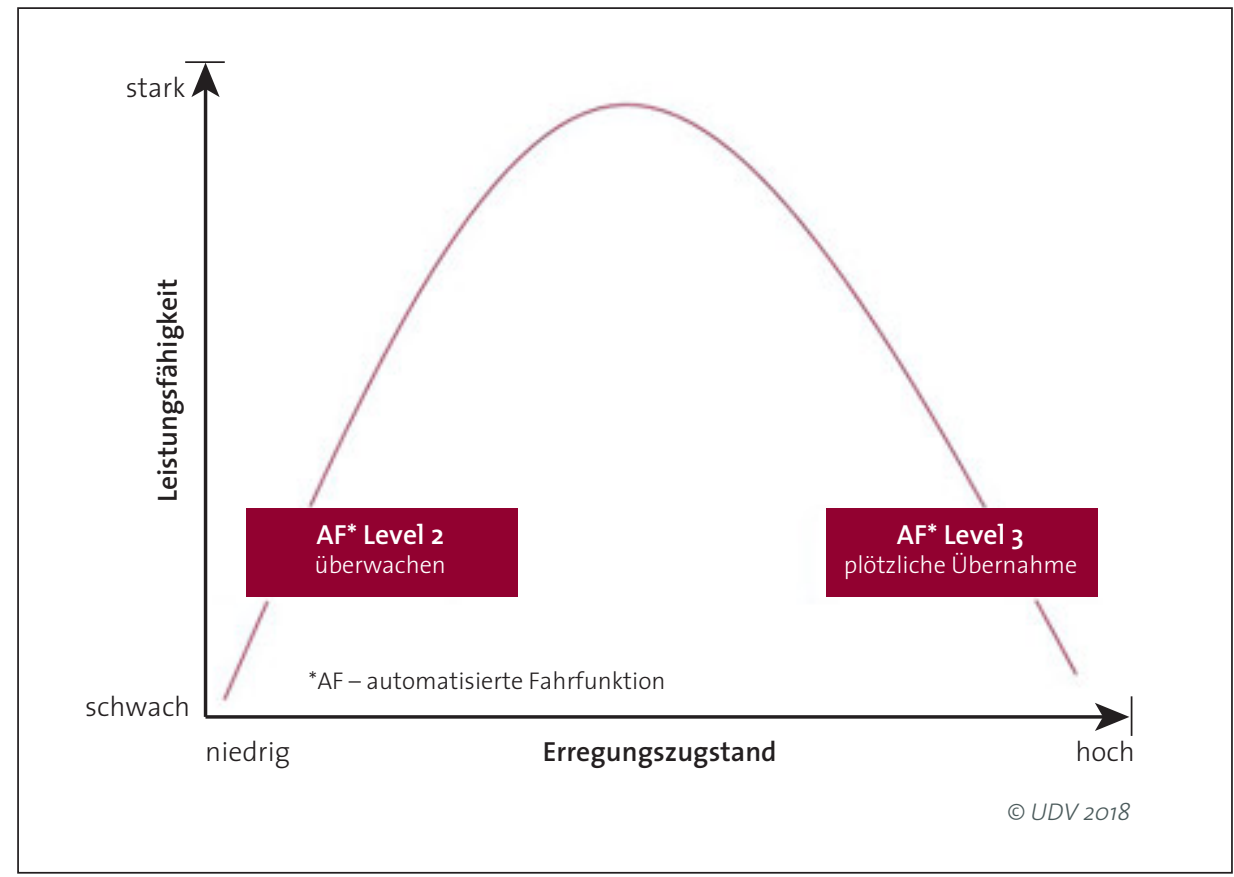

Abb. 4: Vereinfachte Darstellung des Yerkes-Dodson-Gesetzes im Zusammenhang mit automatisiertem Fahren [14]

auf einem der geringen Erregung ähnlichen Minimum zu enden (Abb.4). Mit einfachen Worten ausgedrückt: Der Mensch ist am besten, wenn er eine anspruchsvolle Aufgabe mittlerer Erregung ausführt. Das Führen eines Fahrzeugs ist so eine Aufgabe. Monotone Aufgaben, die auch beim Autofahren auftreten können, wenn man z. B. einer schnurgeraden Straße ohne Verkehr folgt, führen zu geringerer Leistungsfähigkeit oder Versagen. Das Überwachen eines Autobahnpiloten des Levels 2 wäre so eine Aufgabe. Wiederum führt Überforderung des Fahrers auch zu geringer Leistungsfähigkeit bis hin zum Versagen. Die plötzliche Aufforderung zur Übernahme der Fahrfunktion des Autobahnpiloten des Levels 3 kann ein Beispiel dafür sein.
Nach dem gegenwärtigen Stand der Gespräche zu einer UNECE-Regelung über den technischen Standard teilautomatisierter Systeme soll der Fahrer für die sichere Übernahme der Fahraufgabe gegebenenfalls nur vier Sekunden Zeit erhalten. Neueste Forschungsergebnisse der TU Braunschweig zeigen allerdings, dass ein ausgeschlafener Fahrer, wenn er eine beanspruchende Nebenaufgabe ausführt, selbst nach einer kurzen Fahrstrecke mit einem hochautomatisierten System rund acht Sekunden benötigt, um das Fahrzeug zu übernehmen und rund 14 Sekunden, um wieder die volle Situationskontrolle zu erlangen [15]. Bei einem müden Fahrer gilt Vergleichbares bereits ohne Nebenaufgabe. Zudem zeigt eine weitere Studie der TU Braunschweig, dass die Fahrer im hochautomatisierten Modus deutlich schneller ermüden und deshalb eine automatisierte Fahrt (Level 2- und Level 3-Systeme) nicht zu empfehlen ist, die länger als 15 Minuten dauert, wenn der Fahrer kurzfristig die Fahraufgabe übernehmen soll [16]. 


\begin{tabular}{|c|c|c|}
\hline & Manuell / Assistiert & Automatisiert \\
\hline $\begin{array}{c}1 \\
\text { Benennung }\end{array}$ & $\begin{array}{l}\text { Der Name oder die Beschreibung des } \\
\text { Systems muss eindeutig sein und deutlich } \\
\text { die Systemfunktionalität beschreiben. Er } \\
\text { darf in keiner Weise eine Automatisierung } \\
\text { der Funktion unterstellen. }\end{array}$ & $\begin{array}{l}\text { Der Name oder die Beschreibung des Systems muss } \\
\text { eindeutig sein und deutlich die Systemfunktionalität } \\
\text { beschreiben und den Begriff „automatisiert“ beinhalten. }\end{array}$ \\
\hline (2) & $\begin{array}{l}\text { Das Fahrzeugverhalten muss der Straßen- } \\
\text { verkehrsordnung entsprechen. }\end{array}$ & $\begin{array}{l}\text { Während des Betriebes muss sich das System an die } \\
\text { lokalen Verkehrsgesetze halten, einschließlich die ange- } \\
\text { zeigten Geschwindigkeiten befolgen und den Sicher- } \\
\text { heitsabstand zum Vorausfahrenden einhalten. Das } \\
\text { Fahrzeugverhalten muss der Straßenverkehrsordnung } \\
\text { entsprechen. }\end{array}$ \\
\hline $\begin{array}{l}3 \\
\text { Ortsgenau }\end{array}$ & & $\begin{array}{l}\text { Jede Funktion darf nur örtlich begrenzt wirken, um den } \\
\text { Betrieb entsprechend des Auslegungsbereiches sicherzu- } \\
\text { stellen und den Fahrer über die Verfügbarkeit informieren. }\end{array}$ \\
\hline $\begin{array}{l}4 \\
\text { Klare Übergabe }\end{array}$ & $\begin{array}{l}\text { Der Status der Fahrfunktion, manuell } \\
\text { oder assistiert, muss klar und eindeutig } \\
\text { sein, mit klaren Kontrollübergängen. Das } \\
\text { System muss durch das kontinuierliche } \\
\text { Überwachen der Systemfunktion sicher- } \\
\text { stellen, dass der Fahrer die Fahraufgabe } \\
\text { wahrnimmt. }\end{array}$ & $\begin{array}{l}\text { Die Übergabe zum Fahrer und zurück zum System muss } \\
\text { einem Prozess folgen, bei dem das System seine Verfüg- } \\
\text { barkeit anzeigt und der Fahrer die Übernahme bestätigen } \\
\text { muss und umgekehrt. Dies setzt voraus, dass das System } \\
\text { genügend „Sicht voraus“ hat, um im Fall einer fehlgeschla- } \\
\text { genen Übergabe durch das System an den Fahrer sicher } \\
\text { halten zu können (siehe 7). }\end{array}$ \\
\hline Sicheres Fahren & $\begin{array}{l}\text { Der Fahrer muss klar und deutlich auf } \\
\text { seine Verantwortung zum sicheren Fahren } \\
\text { hingewiesen werden und auf seine Verant- } \\
\text { wortung für vorhersehbare Verkehrssitua- } \\
\text { tionen innerhalb des Auslegungsbereiches, } \\
\text { die das System nicht sicher bewältigen } \\
\text { kann. }\end{array}$ & $\begin{array}{l}\text { Wenn sich das System im automatisierten Modus befindet, } \\
\text { muss das Fahrzeug in der Lage sein, alle im Umfeld } \\
\text { des Auslegungsbereiches auftretenden und realistisch } \\
\text { annehmbaren Situationen zu bewältigen. }\end{array}$ \\
\hline $\begin{array}{l}\text { Vermeidung von } \\
\text { Fehlgebrauch }\end{array}$ & $\begin{array}{l}\text { Das Fahrzeug muss erkennen, ob der } \\
\text { Fahrer das System falsch benutzt und } \\
\text { Vorkehrungen zur Vermeidung treffen. } \\
\text { Eine Missachtung von Warnungen sollte } \\
\text { zur Deaktivierung des Systems führen. } \\
\text { Kontinuierlicher Fehlgebrauch muss zum } \\
\text { Abschalten des Systems führen bis zum } \\
\text { nächsten Start des Fahrzeugs. }\end{array}$ & $\begin{array}{l}\text { Das Fahrzeug muss erkennen, ob der Fahrer das System } \\
\text { falsch benutzt und Vorkehrungen zur Vermeidung treffen. }\end{array}$ \\
\hline
\end{tabular}




\begin{tabular}{|c|c|c|}
\hline & Manuell / Assistiert & Automatisiert \\
\hline $\begin{array}{l}\text { Ub } \\
\text { Unerwartete } \\
\text { Übergabe }\end{array}$ & & $\begin{array}{l}\text { Falls das automatisierte Fahrzeug eine Situation erkennt, } \\
\text { die zum Beginn der automatisierten Fahrt nicht vorher- } \\
\text { sehbar war (z.B. widriges Wetter) und dies zu einer früher } \\
\text { als geplanten Übergabe an den Fahrer führt, muss eine } \\
\text { geeignete und angemessene Benachrichtigung erfolgen. }\end{array}$ \\
\hline $\begin{array}{c}7 \\
\text { Sicherer Halt }\end{array}$ & & $\begin{array}{l}\text { Falls der Fahrer nicht auf eine Übernahmeaufforderung } \\
\text { reagiert, muss das Fahrzeug ein „Sicherer-Halt-Manöver“ } \\
\text { ausführen und zu einem sicheren Ort steuern, der dem } \\
\text { Auslegungsbereich und den Verkehrsbedingungen ange- } \\
\text { messen ist. }\end{array}$ \\
\hline Notfalleingriff & & $\begin{array}{l}\text { Falls das Fahrzeug eine plötzliche unvorhergesehene } \\
\text { gefährliche Situation detektiert, muss das System ein risi- } \\
\text { kominimierendes Manöver ausführen, um eine Kollision } \\
\text { abzumildern oder zu vermeiden. }\end{array}$ \\
\hline Ersatzsysteme & & $\begin{array}{l}\text { Das System muss im Falle eines Fehlers genügend Redun- } \\
\text { danzen besitzen, um entweder im automatisierten Modus } \\
\text { weiterzufahren oder eine geplante Übergabe an den Fahrer } \\
\text { zu ermöglichen. Jeder Fehler muss dem Fahrer angezeigt } \\
\text { werden und - wenn angemessen- die Nutzung des Systems } \\
\text { unterbunden oder eingeschränkt werden, bis der Fehler } \\
\text { behoben ist. Laut Definition muss das System eigendiag- } \\
\text { nosefähig sein, um Fehler zu erkennen. Ergänzend muss } \\
\text { das System selbstkalibrierend sein. }\end{array}$ \\
\hline $\begin{array}{l}\text { (10) } \\
\text { Unfalldaten }\end{array}$ & & $\begin{array}{l}\text { Daten zur Unfallaufklärung müssen im Falle einer Kollision } \\
\text { aufgezeichnet werden und gleichberechtigt Herstellern } \\
\text { und berechtigten Dritten zur Verfügung gestellt werden. } \\
\text { So sind Fragen zum Status des automatisierten Systems, } \\
\text { zum Umfang der Fahrereingaben und Haftung schnell und } \\
\text { unabhängig bewertbar. }\end{array}$ \\
\hline
\end{tabular}




\section{Technische Anforderungen an Automatisierungsfunktionen}

Ausgehend von einem klaren Verständnis der Möglichkeiten und Grenzen von Automatisierungsfunktionen sollen Anforderungen an konkrete verkehrssicherheitsrelevante Aspekte dieser Systeme beschrieben werden (Tab. 4). Dabei dient eine klare Kommunikation der Funktionsweise und Leistungsfähigkeit dazu, eine Verwirrung des Fahrers zu vermeiden.

Wie bereits dargestellt, werden auch Fahrzeuge mit Automatisierungsfunktionen im Verkehrsgeschehen verunfallen. Es ist dabei wesentlich, die Fahrzeuge mit den entsprechenden automatisierten Fahrfunktionen identifizieren zu können und autorisierten Dritten einen standardisierten Datenzugang zu ermöglichen, um den Hergang und die Verantwortlichkeiten bei einem Unfall möglichst genau ermitteln zu können. Der Datenumfang sollte dazu aus Sicht der Verkehrssicherheit mindestens beinhalten:

- GPS-Ereignis: Ort und Zeit des Ereignisses

- Automatisierter Status - an oder aus

- Automatisierter Modus - Parken oder Fahren

- Automatisierter Übergang: Zeitstempel

- Aufzeichnung des Fahrereingriffs: Lenken, bremsen, beschleunigen, blinken

- Zeit seit letztem Fahrereingriff

- Fahrersitzbelegung und Fahrerstatus

- Fahrergurt geschlossen

Nur so wird es in Zukunft möglich sein, die Verkehrsunfallforschung zu befähigen, die Unfallursachen unter Beteiligung von Fahrzeugen mit automatisierten Fahrfunktionen zu ermitteln und die richtigen Schlüsse für die Weiterentwicklung der Systeme zu ziehen.

\section{Fazit}

Vollautomatisiert und autonom fahrende Fahrzeuge können ein großer Gewinn für die Verkehrssicherheit sein, wenn sie unter allen Bedingungen in ihrem Auslegungsbereich fehlerfrei funktionieren. Bis diese Systeme auf den Markt kommen, sollte der Fahrer aus Sicht der Verkehrssicherheit in manuell gesteuerten Fahrzeugen durch immer besser werdende Assistenzsysteme bei der Fahraufgabe unterstützt werden. Trotzdem werden in dieser Übergangsphase automatisierte Fahrfunktionen angeboten werden, die die Unterstützung des Fahrers benötigen. Diese sollten die hier vorgestellten Schlüsselmerkmale erfüllen, um kein Verkehrssicherheitsrisiko darzustellen. Grundlegend sollte für alle Fahrzeuge mit automatisierten Fahrfunktionen gelten, dass sie nur im öffentlichen Straßenverkehr zur Anwendung kommen, wenn sie nicht unsicherer sind, als entsprechende mit Fahrerassistenzsystemen ausgerüstete Fahrzeuge. 
Literatur

\section{Literatur}

[1] SAE International:Taxonomy and Definitions for Terms Related to Driving Automation Systems for On-Road Motor Vehicles. SAE Standard J 3016, überarbeitete Version September 2016.

[2] Donges, E.: „Fahrerhaltensmodelle“ in Handbuch Fahrerassistenzsysteme. Herausgeber: Winner, Hakuli, Lotz, Singer, 3. Auflage, Springer Fachmedien, Wiesbaden 2015.

[3] Gasser, T.M., Frey, A. Th., Seeck, A., Auerswald, R.: „Comprehensive Definitions for Automated Driving and ADAS“. 25. ESV-Konferenz, Detroit, 5--9.6.2017.

[4] Verband der Deutschen Automobilindustrie: Automatisierung - Von Fahrerassistenzsystemen zum automatisierten Fahren. VDA Magazin, Berlin 2015.

[5] Statistisches Bundesamt: 0,9\% weniger Verkehrstote im Jahr 2017. Pressemitteilung Nr. 063 vom 27.02.2018

[6] Statistisches Bundesamt: Fachserie 7, Reihe 8, 2016; DESTATIS 2017.

[7] Hummel, H., Kühn, M., Bende, J., Lang, A.: Fahrerassistenzsysteme - Ermittlung des Sicherheitspotentials auf Basis des Schadengeschehens der Deutschen Versicherer. Forschungsbericht FSO3, Berlin 2011.

[8] European New Car Assessment (EuroNCAP): Programm 2020 ROADMAP EUROPEAN NEW CAR ASSESSMENT PROGRAMME, June 2014.

[9] European Commission: Public Consultation on the revision of the Vehicle General Safety Regulation and the Pedestrian Safety Regulation - Background document, EC Directorate-General for Internal Market, Industry, Entrepreneurship and SMEs, Brüssel, 2017.
[10] Unfallforschung der Versicherer (UDV): Verkehrssicherheitspotential einer hochautomatisierten Fahrfunktion (HAF) für Pkw und Lkw, unveröffentlicht, Berlin 2017. [11] Gesamtverband der Deutschen Versicherer (GDV): Automatisiertes Fahren - Auswirkungen auf den Schadenaufwand bis 2035. Abschlussbericht, Berlin 2017. [12] Kühn, M.: Eigene Berechnungen nach Statistisches Bundesamt, Fachserie 7, Reihe 8, 2013 und Verkehr in Zahlen 2014, unveröffentlicht, Berlin 2015.

[13] Klingner, M., Erbsmehl, Ch., Landgraf, T.: Sicherheit des autonomen Fahrens, Zeitschrift für Verkehrssicherheit 63, Nr. 5, S. 244, 2017.

[14] Yerkes, R.M. \& Dodson, J.D. (1908). The relation of strength of stimulus to rapidity of habit-formation. Journal of Comparative Neurology and Psychology, 18, 459-482.

[15] Vogelpohl, T., Vollrath, M., Kühn, M., Hummel, Th., Gehlert, T.: Übergabe von hochautomatisiertem Fahren zu manueller Steuerung - Teil 1: Review der Literatur und Studie zu Übernahmezeiten. Forschungsbericht Nr. 39 , Unfallforschung der Versicherer, Berlin 2016.

[16] Vogelpohl, T., Vollrath, M., Kühn, M.: Übergabe von hochautomatisiertem Fahren zu manueller Steuerung - Teil 2: Müdigkeit und lange Fahrtdauer als Einflussfaktoren auf die Sicherheit nach einer Übernahmeaufforderung. Forschungsbericht Nr. 47, Unfallforschung der Versicherer, Berlin 2017.

[17] Unfallforschung der Versicherer (UDV): Verkehrssicherheit und automatisiertes Fahren, Positionspapier, unveröffentlicht, Berlin 2018. 
18 Unfallforschung kompakt 78 | Wie automatisiertes Fahren die Verkehrssicherheit erhöht

Notizen 


\section{Unfallforschung der Versicherer

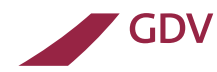

Gesamtverband der Deutschen

Versicherungswirtschaft e.V.

Wilhelmstraße 43/43 G, 10117 Berlin

Postfach 0802 64, 10002 Berlin

Telefon $030.2020-5821$

Fax $030.2020-6633$

unfallforschung@gdv.de

www.udv.de

www.gdv.de

Facebook: facebook.com/unfallforschung

Twitter: @unfallforschung

YouTube: youtube.com/unfallforschung

Instagram: instagram.com/udv_unfallforschung

Redaktion:

Dr.-Ing. Matthias Kühn

Gestaltung:

pensiero KG, wWw.pensiero.eu

Bildquellen:

Titelbild: Uli-B_Fotolia

Erschienen: 08/2018 


\section{GDV}

DIE DEUTSCHEN VERSICHERER

Gesamtverband der Deutschen Versicherungswirtschaft e.V.

Wilhelmstraße 43 / 43G, 10117 Berlin

Postfach 080264,10002 Berlin

Tel.: 030/20 $20-50$ oo, Fax: 030/2020-6000

www.gdv.de, www.udv.de 